VoL. 50 (1994) [41-48]

\title{
ON A THEOREM OF HUGHES AND THOMPSON
}

\author{
R.A. BRYCE
}

We generalise the notion of proper Hughes subgroup in a group, asking that, for some prime $p$, the set-complement of the union of two proper subgroups consist solely of elements of order $p$. A structure theorem for non-p-groups of this type is proved.

\section{INTRODUCTION}

Let $G$ be a group and $p$ a prime. Define the Hughes subgroup $H_{p}(G)$ of $G$ as that generated by the elements of $G$ whose order is not $p$. In [3] Hughes asked if the index of $H_{p}(G)$ in $G$ is necessarily $1,|G|$ or $p$. The question received a positive answer for finite non-p-groups in the article [4] of Hughes and Thompson; and a negative answer for groups in general in Wall [8]. Khukhro [6] has shown that for finite groups the answer is yes 'almost always'. Hughes and Thompson [4] and Kegel [5] prove more: in a finite group $G, H_{p}(G)$ if proper, is nilpotent; their results may be formulated as follows.

Theorem 1. (Hughes and Thompson [4]; Kegel [5]) Let $G$ be a finite group and $p$ a prime. Suppose that $H$ is a proper subgroup of $G$ whose set-complement in $G$ consists of elements of order $p$. Then $H$ is nilpotent. If $G$ is not a p-group then $H$ is normal and of index $p$ in $G$.

A partial generalisation of this result is proved by Espuelas [2]. If $G$ is a finite soluble group, $p$ an odd prime, and $H$ a proper subgroup of $G$ whose complement consists of elements of order at most $p^{n}$, then $B$ has nilpotent length at most $n$.

Another direction of generalisation is taken in [1]. There it is shown that a group $G$ of exponent $p^{n}$, soluble of derived length $d$ and in which $H_{p}(G)$ is proper, is nilpotent with class bounded by a function of $p, n, d$.

Yet another generalisation is proposed here. Suppose that a group $G$ has subgroups $A_{i}(i \in I)$ the set-complement of whose union consists of elements of order $p$. What can we say about the structure of $G$ ? What hope is there of generalising Theorem 1 ?

We present results on this problem for the case when $|I|=2$, and raise some questions. We show that in this case it is possible to characterise the groups concerned,

Received 20th September, 1993

Copyright Clearance Centre, Inc. Serial-fee code: 0004-9729/94 \$A2.00+0.00. 
as it is in the Hughes-Thompson context, and in much the same way. The methods presented here do not seem to generalise; in a later joint article with Professors V. Fedri and L. Serena, completely different methods will be used to get results for finite groups in the general case. These results are, however, less complete in general than those presented here.

\section{The Classes $\mathcal{H}_{n}(p)$}

Let $p$ be a prime and $n$ a positive integer. Define the class $\mathcal{H}_{n}(p)$ to consist of all finite groups $G$ with subgroups $A_{i}(1 \leqslant i \leqslant n)$ whose set-union is not $G$, and the complement of whose set-union consists of elements of order $p$. It will be convenient to include the identity group in $\mathcal{H}_{n}(p)$. The groups with proper Hughes subgroup for exponent $p$ comprise the class $\mathcal{H}_{1}(p)$. It is easy to construct groups in $\mathcal{H}_{n}(p)$ in general. For example, suppose that $G_{i}(1 \leqslant i \leqslant n)$ are groups in which $H_{p}\left(G_{i}\right) \neq$ $G_{i}(1 \leqslant i \leqslant n)$. Consider the direct product $G=\Pi\left(G_{i}: 1 \leqslant i \leqslant n\right)$, and its subgroups $A_{i}=\Pi\left(G_{j}: j \neq i\right) \times H_{p}\left(G_{i}\right)(1 \leqslant i \leqslant n)$. Then $G \neq \cup\left(A_{i}: 1 \leqslant i \leqslant n\right)$, and every element of $G$ outside this union has order $p$ since it projects outside $H_{p}\left(G_{i}\right)$ for every $i$. In particular, for example, if $S_{3}$ denotes the symmetric group on three symbols, then $S_{3} \times S_{3}$ is in $\mathcal{H}_{2}(2)$. However, since $S_{3} \times S_{3}$ is generated by elements of order six, $S_{3} \times S_{3}$ is not in $\mathcal{H}_{1}(2)$. By definition $\mathcal{H}_{m}(p) \subseteq \mathcal{H}_{n}(p)$ for $m \leqslant n$ and we may ask if the inclusion $\mathcal{H}_{m}(p) \subseteq \mathcal{H}_{n}(p)(m<n)$ is always strict. This question is not further prosecuted in this article.

Another interesting question concerns the structure of groups in $\mathcal{H}_{n}(p)$. Do they necessarily have the structure of the example given above? This is not true in general. For example, $S_{3}$ lies in $\mathcal{H}_{3}(3)$. (Note also that $S_{3}$ lies in $\mathcal{H}_{1}(2)$, so the prime $p$ chosen does matter.) A consequence of Theorem 1 is that the groups in $\mathcal{H}_{1}(p)$ are soluble. In general groups in $\mathcal{H}_{n}(p)$ are not soluble. For example, a group in which the Sylow $p$-subgroups are of order $p$ and self-centralising is necessarily in $\mathcal{H}_{n}(p)$ for some $n$ : the subgroups $A_{i}$ could be chosen as the cyclic subgroups whose order is not $p$. An example is $A_{5}$, the alternating group on five symbols, and we can be more economical: every element of $A_{5}$ not of order five is contained in a copy of $A_{4}$ of which there are five in number. Hence $A_{5}$ is in $\mathcal{H}_{5}(5)$. Nevertheless we shall prove that in the case $n$ $=2$ the questions raised here do have positive answers.

We include in this section some elementary, useful results needed later. All groups considered in this paper are finite.

LEMMA 1. Suppose that a group $G$ has subgroups $A_{i}(1 \leqslant i \leqslant n)$ whose union is not $G$ and the complement of whose union consists of elements of order p. Let $D=$ $\cap\left(A_{i}: 1 \leqslant i \leqslant n\right)$. Then core $G(D)$ is nilpotent.

Proof: If $N=\operatorname{core}_{G}(D)$, and $g$ is in $G \backslash \cup\left(A_{i}: 1 \leqslant i \leqslant n\right)$, then every element of 
$N\langle g\rangle \backslash N$ is outside $\cup\left(A_{i}: 1 \leqslant i \leqslant n\right)$. Hence it has order $p$. Therefore, by Theorem $1, N$ is nilpotent.

LEMma 2. Let $G$ be a group and $\mathcal{A}$ a union of subgroups of $G$. Suppose that $\mathcal{A} \neq G$ and that, for some prime $p$, every element of $G \backslash \mathcal{A}$ has order $p$. Then ${ }^{*} C_{G}(x)$ is a $p$-group for all $x \in G \backslash \mathcal{A}$.

Proof: If $y$ is a $p^{\prime}$-element of $C_{G}(x)$ then $x y$ is not of order $p$ and therefore $x y \in \mathcal{A}$. Since $\mathcal{A}$ is a union of groups it follows that $x \in \mathcal{A}$, a contradiction. Hence $C_{G}(x)$ is a $p$-group.

A corollary of this is that nilpotent groups in $\mathcal{H}_{n}(p)$ are $p$-groups.

In section 4 we need the idea of a Frobenius group, one admitting a fixed-pointfree automorphism of order $p$. Such a group is necessarily nilpotent, by a theorem of Thompson [7]. It is, moreover, a $\boldsymbol{p}^{\prime}$-group, since otherwise the automorphism normalises a Sylow $p$-subgroup of the group, therefore centralising some non-trivial element. By a Frobenius extension we mean a group $H\langle s\rangle$ where $H$ is a group and $s$ a fixed-point-free automorphism of it of prime order. The next lemma, though formulated in the language of the present context, is well known, and is included for completeness only.

LEMma 3. Let $H$ be a nilpotent $\boldsymbol{p}^{\prime}$-group admitting an automorphism $s$ of order p. Write $G=H\langle s\rangle$. Then $G$ is in $\mathcal{H}_{1}(p)$ if and only if $\mathbf{C}_{H}(s)=1$.

Proof: That the condition $C_{H}(s)=1$ is necessary follows from Lemma 2.

Conversely suppose that $\mathbf{C}_{H}(s)=1$. For all $h \in H$ we show that $(s h)^{p}=1$. Now $(s h)^{p}=h^{s^{p-1}} h^{s^{p-2}} \ldots h^{s} h$. If $H$ is Abelian this element is fixed by $s$, so $(s h)^{p}=1$. If $H$ is not Abelian we deduce, by induction on the class of $H$, that the element displayed above is in $\zeta_{1}(H)$. It is centralised by $s h$, and therefore by $s$, and is therefore 1 . That is $(s h)^{p}=1$ for all $h \in H$. Finally note that, for $i \in(1,2, \ldots, p-1), C_{H}\left(s^{i}\right)=$ $C_{H}(s)=1$ so, for all $h \in H,\left(s^{i} h\right)^{p}=1$. Therefore $H_{1}(p) \subseteq H$ so $G \in \mathcal{H}_{1}(p)$.

\section{INITIAL STRUCTURE THEOREM}

In this short section we give a structure theorem for $\mathcal{H}_{2}(p)$ groups which is used in the next section to delineate their structure more precisely.

THEOREM 2. Let $G$ be a finite group which, for some prime $p$, is not a $p$-group. Suppose that $G$ has proper subgroups $A_{1}, A_{2}$ and that the complement of their union contains only elements of order $p$. Then one of the following holds.

$$
\begin{aligned}
& \text { One of } G \backslash A_{1}, G \backslash A_{2} \text { consists of elements of order } p \text {. } \\
& O_{p^{\prime}}(G) \subseteq A_{1} \cap A_{2} \text { and } G / O_{p^{\prime}}(G) \text { is a p-group. }
\end{aligned}
$$

Proof: Choose a pair $A, B$ of subgroups of $G$ with the properties that $A \subseteq$ $A_{1}, B \subseteq A_{2}$; that $G \backslash(A \cup B)$ consists of elements of order $p$; and that, subject to these 
constraints, $(A, B)$ is minimal in the component-wise ordering of pairs of subgroups of $G$. With this choice

$A, B$ normalise each other.

For, if $b$ is in $B$ then the complement of $A^{b} \cup B=(A \cup B)^{b}$ consists of elements of order $p$. Moreover $\left(A^{b} \cap A\right) \cup B=\left(A^{b} \cup B\right) \cap(A \cup B)$, so every element in the complement of the left-side has order $p$ since it fails to lie in a term of the right-side. By minimality $A=A^{b} \cap A$ whence $A \subseteq A^{b}$ and therefore $A=A^{b}$. Since this holds for all $b$ in $B, B$ normalises $A$. Similarly $A$ normalises $B$.

Suppose that (3.1) does not hold. That is, neither $G \backslash A_{1}$ nor $G \backslash A_{2}$ consists of elements of order $p$. The same is therefore true of $G \backslash A$ and $G \backslash B$. Hence, in particular, $A, B$ are incomparable. Write $D=A \cap B$. Then, by (3.3), $D$ is normal in $A B$. Moreover if $x$ is not in $A B$ then $x^{p}=1$. Hence, by Theorem $1, A B$ is normal in $G$. Let $a, b$ be elements of $A \backslash D$ and $B \backslash D$ respectively. Then $a b$ lies outside $A \cup B$ and therefore has order $p$. Hence $1=(a b)^{p}=a^{p} b^{p}(\bmod D)$ and so $a^{-p}=b^{p}(\bmod D)$, which means that

$$
A / D, B / D \text { have exponent } p \text {. }
$$

That is, $D$ is subnormal of $p$-power index in $G$. Hence all $p^{\prime}$-elements of $G$ must lie in $D$. Also every element of $D\langle a b\rangle \backslash D$ has order $p$, so $D$ is nilpotent, by Theorem 1. It follows that $O_{p^{\prime}}(G)=O_{p^{\prime}}(D)$ and $G / O_{p^{\prime}}(G)$ is a $p$-group. This completes the proof of Theorem 2.

The properties of a minimal pair $(A, B)$ of subgroups introduced in this proof will be used again in the next section.

\section{Finer structure of $\mathcal{H}_{2}(p)$-GRoups}

Theorem 1 reduces the classification of groups in $\mathcal{H}_{1}(p)$ to the following two problems: firstly, classify $p$-groups in $\mathcal{H}_{1}(p)$; secondly, classify Frobenius extensions. An $\mathcal{H}_{1}(p)$-group is a special type of subdirect product of a $p$-group in $\mathcal{H}_{1}(p)$ and a Frobenius extension.

Theorem 3. A finite non-p-group is in $\mathcal{H}_{1}(p)$ if and only if $G=P N$ where $P$ is a $p$-group, $N$ a $p^{\prime}$-group normal in $G$, and where:

$$
\begin{aligned}
& C_{N}(P)=1 \\
& H_{p}(P) \subseteq C_{P}(N) \\
& \left|P: C_{P}(N)\right|=p .
\end{aligned}
$$


In particular a group in $\mathcal{H}_{1}(p)$ is a subdirect product of a $p$-group in $\mathcal{H}_{1}(p)$ and a Frobenius extension.

Proof: That non-p-groups in $\mathcal{H}_{1}(p)$ have this structure is a consequence of Theorem 1. For, let $P$ be a Sylow $p$-group of a group $G$ in $\mathcal{H}_{1}(p)$, and let $N=O_{p^{\prime}}(G)$. Then $G=P N$. Now let $y \in P \backslash H_{p}(G)$. Firstly, all such $y$ have order $p$ and hence $H_{p}(P) \subseteq P \cap H_{p}(G) \neq P$ so that $P \in \mathcal{H}_{1}(p)$. Secondly, $y$ has no non-trivial fixed points in $N$ by Lemma 2, so $C_{N}(P)=1$. Also, using Theorem 1 and Lemma 2, $P \cap H_{p}(G) \subseteq C_{P}(N) \subseteq P \cap H_{p}(G)$ so $C_{P}(N)=P \cap H_{p}(G)$, and hence $\left|P: C_{P}(N)\right|=p$. We see also that $G$ is a subdirect product of $P$, a $p$-group in $\mathcal{H}_{1}(p)$, and $G / P \cap H_{p}(G)$, a Frobenius extension.

Conversely, if $G$ has the structure described, let $x \in G \backslash C_{P}(N) N$. Then $x=y n$ where $y \in P \backslash C_{P}(N)$ and $n \in N$. By hypothesis $y^{P}=1$ and $y$ has no fixed points in $N$. Hence $(y n)^{p}=1$ by Lemma 3. Therefore $G \in \mathcal{H}_{1}(p)$.

This completes the proof of Theorem 3 .

We use Theorem 2 in a similar way to describe the groups in $\mathcal{H}_{2}(p)$.

THEOREM 4. A finite non-p-group $G$ is in $\mathcal{H}_{2}(p)$ if and only if it has a $p$-subgroup $P$ and a nilpotent normal $p^{\prime}$-subgroup $N$, with $G=P N$ satisfying the following conditions:

$$
C_{N}(P)=1
$$

$P$ has proper subgroups $P_{1}, P_{2}$ the complement of whose union consists of elements of order $p$;

and either

$$
C_{P}(N)=P_{i} \text { and }\left|P: P_{i}\right|=p \text {, where } i \text { is one of } 1 \text { or } 2 \text {; }
$$

or

$$
N=N_{1} \times N_{2}, P_{i}=C_{P}\left(N_{i}\right) \text { and }\left|P: P_{i}\right|=p(i=1,2) .
$$

Proof: Note first of all that a group in $\mathcal{H}_{1}(p)$ comes under the alternative (4.6). This follows from Theorem 3, with the choice of $P_{2}$, say, as 1 .

Hence suppose that $G$ is in $\mathcal{H}_{2}(p)$ but not in $\mathcal{H}_{1}(p)$. There are in $G$ subgroups $A_{1}, A_{2}$ whose union is not $G$, and the complement of whose union consists of elements of order $p$. If $A, B$ are chosen minimally, as in the proof of Theorem 2, then $A B=G$ (or else $G$ would be in $\mathcal{H}_{1}(p)$ ), $A, B$ are normal in $G$, and they are incomparable. Write $D=A \cap B$.

Write $N=O_{p^{\prime}}(G)$. Let $P$ be a Sylow $p$-subgroup of $G$. Then, by Theorem 2, $G=P N$. Since $P$ is not contained in $A \cup B$ it follows from Lemma 2 that $C_{N}(P)=1$ 
confirming (4.4). Write $P_{1}=P \cap A_{1}$ and $P_{2}=P \cap A_{2}$; both are proper and the complement in $P$ of their union consists of elements of order $p$. This confirms (4.5).

Let $H / K$ be a chief factor of $G$ contained in $N$, and write $C=C_{G}(B / K)$. It must be that $D \subseteq C$ by Lemma 1 , since $D \unlhd G$. Moreover $C \subseteq A \cup B$ by Lemma 2. Since $C$ is not a union of two proper subgroups we have either $C \subseteq A$ or $C \subseteq B$. It cannot be that $C$ is contained in $D$, as otherwise $C=D$, and $G / C$ is a faithfully represented $p$-group with non-cyclic centre. So suppose that $D \subset C \subseteq B$. Now $G$ acts on $H / K$ with kernel $C$, that is as $A / D \times B / C$. However a faithfully and irreducibly represented nilpotent group has cyclic centre, so it follows that $B / C$ is trivial, since $A / D$ is not. That is, $C=B$.

We have proved that the centraliser of a chief factor of $G$ contained in $N$ is either $A$ or $B$. If $C_{0}=C_{P}(N)$ then $N C_{0}$ centralisers every chief factor of $G$ in $N$. If $N C_{0}$ is not contained in $D$ therefore, $N C_{0}$ is in $A$ or in $B$, say in $B$. However if $C$ is the centraliser of some chief factor of $G$ in $N$, then we have $D \subset N C_{0} \subseteq C \subseteq B$. It follows that $C=B$, and this for every $p^{\prime}$-chief factor centraliser. Now

$$
\begin{aligned}
B & =\cap\left(C_{G}(H / K): H / K \text { a } p^{\prime} \text {-chief factor }\right) \\
& =\cap\left(N C_{P}(H / K): H / K \text { a } p^{\prime} \text {-chief factor }\right) \\
& =N\left[\cap\left(C_{P}(H / K): H / K \text { a } p^{\prime} \text {-chief factor }\right)\right] \\
& =N C_{0} .
\end{aligned}
$$

We deduce also that $A / D$ acts fixed-point-freely on each chief factor in $N$ since, for every $a \in A \backslash D$, and for $b \in B \backslash D, a b \notin A \cup B$ so it must act fixed-point-freely on $N$, and therefore fixed-point-freely on every chief factor in $N$. Therefore, since $A / D$ has exponent $p$ by (3.4), $A / D$ is of order $p$. One consequence of this is that $B$ has index $p$ in $G$, and hence $B=A_{2}$. Also $N C_{0}=A_{2}$. Hence $P_{2}=A_{2} \cap P=N C_{0} \cap P=C_{P}(N)$. This takes care of (4.6).

There remains the possibility that $C_{0} \subseteq D$. Then $N C_{0}=D$. Moreover the kernel of the action of $G$ on a chief factor $B / K$ in $N$ is either $A$ or $B$, and $A / D$ or $B / D$ acts faithfully and fixed-point-freely on $H / K$, as the case may be. Furthermore there is at least one such chief factor not centralised by $A$, and at least one not centralised by $B$. Therefore $A / D, B / D$ are both of order $p$. Hence both $A, B$ are of index $p$ in $G$, in fact $A=A_{1}, B=A_{2}$; so both $P_{1}, P_{2}$ are normal and have index $p$ in $P$. Note that $C_{P}(N)=P_{1} \cap P_{2}$, and $P=P_{1} P_{2}$.

Let $N_{1}=C_{N}\left(P_{1}\right), \quad N_{2}=C_{N}\left(P_{2}\right)$. Then $N=N_{1} \times N_{2}$. This is because, firstly, $N=N_{1} \times\left[N, P_{1}\right]$, since $P_{1}$ acts on $N$ as a group of co-prime order; secondly $\left[N, P_{1}, P_{2}\right]=\left[N, P_{2}, P_{1}\right]$, since $P_{1}, P_{2}$ commute in their action on $N$; thirdly $\left[N, P_{1}, P_{2}, \ldots, P_{1}, P_{2}\right]=1$ for sufficiently many entries $P_{1}, P_{2}$ since one 
of $P_{1}, P_{2}$ centralises each chief factor of $G$ in $N$; and fourthly, therefore, $1=$ $\left[N, P_{1}, \ldots, P_{1}, P_{2}, \ldots, P_{2}\right]=\left[N, P_{1}, P_{2}\right]$. Hence $\left[N, P_{1}\right] \subseteq C_{N}\left(P_{2}\right)=N_{2}$, so $N=$ $N_{1} N_{2}=N_{1} \times N_{2}$ since $N_{1} \cap N_{2} \subseteq C_{N}(P)=1$. Finally note that $C_{P}\left(N_{i}\right)=P_{i}(i=1,2)$ since $P_{i} \subseteq C_{P}\left(N_{i}\right) \neq P$.

Conversely suppose that a group $G$ satisfies the conditions of Theorem 4 . Let $A_{i}=P_{i} N(i=1,2)$. Both are proper subgroups of $G$, so their union is not $G$. Consider first the case (4.6). Let $x \in P \backslash\left(P_{1} \cup P_{2}\right)$. Then $x$ has no non-trival fixed points in $N$, because of (4.4). Hence, by Lemma 3 , every element of the form $x n\left(x \in P \backslash\left(P_{1} \cup P_{2}\right), n \in N\right)$ has order $p$. However all elements of $G$ not in $A_{1} \cup A_{2}$ are of this form. Therefore $G \in \mathcal{H}_{2}(p)$.

Finally suppose that $G$ satisfies (4.7). If $P_{1}=P_{2}$ then the conditions of Theorem 3 are satisfied with $P_{1} N=H_{p}(G)$, and so $G \in \mathcal{H}_{1}(p)$. So suppose that $P_{1} \neq P_{2}$. Every element of $G$ not in $A_{1} \cup A_{2}$ has the form $y=x_{1} x_{2} n$ where $x_{1}, x_{2}$ are in $P \backslash P_{1}$ and $P \backslash P_{2}$ respectively, and $n$ is in $N$. However $x_{1} x_{2} \notin P_{1} \cup P_{2}$ and so has no fixed points in $N$ by (4.4). By Lemma 3 therefore, $y$ has order $p$. This means that $G$ is in $\mathcal{H}_{2}(p)$.

The proof of Theorem 4 is complete.

Corollary. A group in $\mathcal{H}_{2}(p)$ is a sub-direct product of a p-group in $\mathcal{H}_{2}(p)$ and either one or two Frobenius extensions.

Proof: This follows at once from Theorem 4. The case (4.6) involves one Frobenius extension, the case (4.7), two.

It will be observed that the examples discussed in section 2 are, in the case $n=2$, special cases of the structure described by Theorem 4 .

\section{REFERENCES}

[1] R.A. Bryce, 'The subgroups of Baer and Hughes', Arch. Math. 61 (1993), 305-312.

[2] A. Espuelas, 'The Fitting length of the Hughes subgroup', J. Algebra 105 (1987), 365-371.

[3] D.R. Hughes, 'Research Problem No. 3', Bull. Amer. Math. Soc. 63 (1957), p. 209.

[4] D.R. Hughes and J.G. Thompson, 'The $H_{p}$-problem and the structure of $H_{p}$-groups', Pacific J. Math. 9 (1959), 1097-1101.

[5] O. Kegel, 'Die Nilpotenz der $H_{p}$-Gruppen', Math. $Z$. 75 (1961), 373-376.

[6] E.J. Khukhro, 'On Hughes' problem for finite p-groups', Algebra and Logic 26 (1987), 398-401.

[7] J.G. Thompson, 'Finite groups with fixed point free automorphisms of prime order', Proc. Nat. Acad. Sci. (USA) 45 (1959), 578-581.

[8] G.E. Wall, 'On Hughes' $H_{p}$-problem', in Proc. Internat. Conf. Theory of Groups, Austral. Nat. Univ. Canberra, August 1965 (Gordon and Breach Science Publishers, Inc., 1967), pp. 357-362. 
School of Mathematical Sciences

The Australian National University

Canberra ACT 0200

Australia 